\title{
Structural, Electronic and Magnetic Properties of Bimetallic Sandwich BzVBzTMBz Clusters and Their Infinite Molecular Wires $[\mathrm{BzVBzTM}]_{\infty}$
}

\author{
Xiuyun Zhang ${ }^{*}$, Tong Zhang, and Yongjun Liu \\ Department of Physics, Yangzhou University, Yangzhou, 225009, China \\ *Correspondence author, E-mail: xyzhang@yzu.edu.cn
}

doi:10.5618/chem.2011.v2.n1.4 || Received: 27-06-2012, Accepted: 19-08-2012, Available online: 20-08-2012

\begin{abstract}
We systematically investigate the stability, electronic and magnetic properties of bimetallic benzene-ligand organic sandwich clusters, BzVBzTMBz $\left(\mathrm{TM}=\mathrm{Sc}, \mathrm{Ti}, \mathrm{Cr}-\mathrm{Ni}, \mathrm{Bz}=\mathrm{C}_{6} \mathrm{H}_{6}\right)$ and their one-dimensional infinite molecular wires, $[\text { BzVBzTM }]_{\infty}$, by employing spin-polarized density functional theory calculations. The BzVBzTMBz clusters adopt normal sandwich conformations with $\mathrm{C}_{6 \mathrm{~V}}$ symmetry except $\mathrm{TM}=\mathrm{Co}$ and $\mathrm{Ni}$. Most BzVBzTMBz clusters are ferromagnetic with BzVBzTMBz ( $\mathrm{TM}=\mathrm{Cr}, \mathrm{Fe})$ having largest magnetic moment of $3 \mu_{\mathrm{B}}$. More interestingly, onedimensional $[\mathrm{BzVBzTM}]_{\infty}(\mathrm{TM}=\mathrm{Cr}-\mathrm{Fe})$ wires are found to be robust half metallic ferromagnets. Among which, $[\mathrm{BzVBzTM}]_{\infty}(\mathrm{TM}=\mathrm{Cr}$, Mn) possess the magnetic moments as high as $5 \mu_{B}$ and $4 \mu_{B}$ per unit cell, respectively. Furthermore, we find that the covalent $T M-B z$ interaction enhances the half-metallicity or metallicity in $\left[\mathrm{BzTM}_{1} \mathrm{BzTM}_{2}\right]_{\infty}$ as compared to the $\left[\mathrm{CpTM}_{1} \mathrm{CpTM}_{2}\right]_{\infty} \quad(\mathrm{Cp}=$ cyclopentyle) analogues which ionic TM-Cp interaction are dominant.
\end{abstract}

Keywords: density functional theory; sandwich clusters and nanowires; ferromagnetic; half metallic

\section{Introduction}

Metal-ligand complexes have received considerable attention over the past decades because of their rich structural, physical and chemical properties and potential applications in catalysis, polymers and molecular recognition, to name a few [1-20]. One typical example is vanadium-benzene, $\mathrm{V}_{\mathrm{n}} \mathrm{Bz}$, whose multidecker structure up to $\mathrm{n}=7$ have been successfully synthesized [18], and the magnetic moments (MMs) increase linearly with cluster size $[6,7,9,10,14,15]$. In addition, one dimensional (1D) $(\mathrm{VBz})_{\infty}$ molecular wire has also been extensively explored and predicted to be ferromagnetic (FM) (quasi) half metal (HM) [10-14]. Similar FM HMs have also been identified for its $1 \mathrm{D}$ analogues,
$(\mathrm{MnBz})_{\infty}$ and $(\mathrm{TMCp})_{\infty}(\mathrm{TM}=\mathrm{V}, \mathrm{Cr}, \mathrm{Mn})$ [19,20]. Moreover, the vanadium-borazine ligand sandwich clusters, $\mathrm{V}_{\mathrm{n}}\left(\mathrm{B}_{3} \mathrm{~N}_{3} \mathrm{H}_{6}\right)_{\mathrm{n}+1}$, were also theoretically reported to be stable ferromagnets, and their 1D infinite $\left[\mathrm{V}\left(\mathrm{B}_{3} \mathrm{~N}_{3} \mathrm{H}_{6}\right)\right]_{\infty}$ wire was also identified to be HM as well $[21,22]$.

On the other hand, bimetallic organic sandwich complexes (BOSCs), composed of organic cyclic molecules and two kinds of metal elements, are also attracting ever-growing interests since their physical and chemical properties can be tailored by different chemical components. The first synthesized multidecker bimetallic organic sandwich complexes are $\mathrm{TM}_{n}\left(\mathrm{FeCp}_{2}\right)_{n+1}(\mathrm{TM}=\mathrm{V}, \mathrm{Ti})$ [23]. First-principle calculation showed that the MMs of 1D bimetallic organic sandwich molecular wires (BOSMWs), $\mathrm{TM}_{n}\left(\mathrm{FeCp}_{2}\right)_{n+1}(\mathrm{TM}=\mathrm{Ti}, \mathrm{V}, \mathrm{Mn})$, are quite large and increase linearly with the cluster size $n$ [24,25]. Examination on the spin-dependent transport properties on $(\mathrm{CpFeCpV})_{n}$ coupled to magnetic $\mathrm{Ni}$ electrodes indicated that they were largely influenced by the contact condition as well as molecular length [26]. A recent DFT calculation shows that most 1D BOSMWs, $\left[\mathrm{CpTM}_{1} \mathrm{CpTM}_{2}\right]_{\infty}, \quad\left(\mathrm{TM}_{1}=\mathrm{Ti}, \mathrm{Cr}, \mathrm{Fe}\right.$, $\mathrm{TM}_{2}=\mathrm{Sc}-\mathrm{Co}$ ) are FM with tunable magnetic moments, and more interestingly, $[\mathrm{CpTiCpTM}]_{\infty}(\mathrm{TM}=\mathrm{Cr}, \mathrm{Fe})$, $[\mathrm{CpCrCpTM}]_{\infty}(\mathrm{TM}=\mathrm{Fe}, \mathrm{Co})$, and $[\mathrm{CpFeCpCo}]_{\infty}$ are robust HMs with large HM gaps [27].

According to the Huckel rule $(4 n+2, n$ is integer), one $\mathrm{Cp}$ radical (five valence electrons) needs to gain one electron to form sable sandwich CpTMCp...Cp alignments [19,25,27]. Differently, Bz molecule has six valence electrons and need not capture extra electrons in BzTMBz...Bz sandwich configurations, thereby, the interaction mechanism inside TM-Bz and TM-Cp should be significantly different [19]. Derived from this logic, the Bz-based BOSCs are expected to show intrinsic differences from the $\mathrm{Cp}$-based ones, in TM-ligand interaction and their resultant electronic and magnetic properties. However, compared with great efforts on $\mathrm{Cp}$-ligand BOSCs, ideal or real benzene-based BOSCs have never been established, either theoretically or experimentally. Therefore, it is 
desirable to explore the structural, electronic and magnetic properties of Bz-based bimetallic sandwich clusters and their infinite molecular wires and address their similarities and differences with the Cp-based analogues.

In this work, based on ab initio calculations, we systematically investigate the structural, electronic and magnetic properties of BzVBzTMBz clusters $(\mathrm{TM}=\mathrm{Sc}, \mathrm{Ti}, \mathrm{Cr}-\mathrm{Ni}$ ) and their 1D BOSMWs, $[\mathrm{BzVBzTM}]_{\infty} \quad(\mathrm{TM}=\mathrm{Sc}, \mathrm{Ti}, \mathrm{Cr}-\mathrm{Fe})$ within the framework of DFT. The reason we choose $\mathrm{BzVBz}$ $\left(\mathrm{VBz}_{2}\right)$ base is that it is very stable among the most explored $\mathrm{TM}_{\mathrm{n}} \mathrm{Bz}_{\mathrm{m}}$ sandwich complexes, and might be analog to the stable $\mathrm{FeCp}_{2}$ base in $\mathrm{TM}_{\mathrm{n}}\left(\mathrm{FeCp}_{2}\right)_{\mathrm{n}+1}$ [23-27]. Besides, the total valence electron number in $\mathrm{VBz}_{2}$ is 17 , short of one electron to satisfy the 18-electron rule, the unsatisfied coordination bond might make the interaction between $\mathrm{VBz}_{2}$ and $\mathrm{TM}$ atoms practically feasible.

\section{Computational Method}

The spin-polarized DFT calculations are implemented in the Vienna $\mathrm{Ab}$ initio Simulation Package (VASP) $[28,29]$. The exchange-correlation potentials are treated by the generalized gradient approximation (GGA) parameterized by Perdew, Burke and Ernzerholf (PBE) [30]. The interaction between valence electrons and ion cores is described by the projected augmented wave (PAW) method [31,32]. The combination of PBE/PAW has been widely used to describe the electronic and magnetic properties of metal-ligand sandwich clusters and wires in literatures [19,26,27].

For the calculations of BzVBzTMBz clusters, we used a simple cubic supercell of $20 \AA \times 20 \AA \times 20 \AA$ to ensure the interaction between the adjacent supercell is negligible, and the reciprocal space is only sampled by the Gamma point. We start from $\mathrm{C}_{6 \mathrm{v}}$ symmetry and fully relax the clusters without any symmetry constraint. To avoid the cluster being trapped in a local-minimum spin state, various spin projection values are assigned to the cluster. Furthermore, the lowest-energy structures are further verified to be true minima via harmonic frequency computations using the DFT-based semicore pseudopotential basis set (DSPP) implemented in DMol3 package [33].

For 1D $[\mathrm{BzVBzTM}]_{\infty}$ wires, periodic boundary condition is applied along the TM-Bz principle axis with the unit cell containing two metal atoms and two $\mathrm{Bz}$ molecules (Fig. S1, red dotted line). In order to explore both the ferromagnetic and antiferromagnetic states, a supercell with two repeating unit cells (Fig. S1, blue dotted line) containing four metal atoms and four $\mathrm{Bz}$ rings labeled as [BzVBzTMBzVBzTM $]_{\infty}$ is used. The vacuum spaces different along TM-Bz axis are set as $20 \AA$. The energy cutoff for the plane wavefunction is $400 \mathrm{eV}$ and the force acting on each atom is less than $0.01 \mathrm{eV} / \AA$. The ions in the periodic unit are allowed to fully relax and the reciprocal space is sampled by $1 \times 1 \times 15$ or $1 \times 1 \times 7$ grid meshes using the Monkhorst-Pack scheme for single-cell and supercell optimization, respectively. And much denser k-point grid $(1 \times 1 \times 45)$ is used for the calculation of the band structures.

\section{Results and Discussion}

\subsection{Finite sandwich BzVBzTMBz $(\mathrm{TM}=\mathrm{Sc}, \mathrm{Ti}$, Cr-Ni) Clusters}

Structural properties The optimized lowest-energy structures of $\mathrm{BzVBzTMBz} \quad(\mathrm{TM}=\mathrm{Sc}, \mathrm{Ti}, \mathrm{Cr}-\mathrm{Ni})$ clusters are displayed in Fig. S2 in Supporting Information, and the structures/symmetries, magnetic moments, energy gaps between HOMO (the highest occupied molecular orbitals) and LUMO (the lowest unoccupied molecular orbitals), distances of TM atoms to the mass centers of $\mathrm{Bz}$ rings, relative energies of the second higher energy spin states respect to the lowest energy spin states of the clusters are presented in Table 1. As shown in Fig. S2, five $\mathrm{BzVBzTMBz}(\mathrm{TM}=\mathrm{Sc}, \mathrm{Ti}, \mathrm{Cr}, \mathrm{Fe}, \mathrm{Mn})$ clusters have eclipsed sandwich conformations with $\mathrm{C}_{6 \mathrm{~V}}$ symmetry. Nevertheless, significant structural distortion are identified for $\mathrm{BzVBzCoBz}$ and $\mathrm{BzVBzNiBz}$, where largely tilted " $\mathrm{CoBz} 2$ " and " $\mathrm{NiBz}_{2}$ " units are found, similar with their corresponding pure $\mathrm{TMBz}_{2}$ clusters $[15,34]$. The $\mathrm{C}-\mathrm{C}$ bond lengths of all the studied clusters are around $1.410 \AA \sim 1.455 \AA$, the $\mathrm{C}-\mathrm{H}$ bond lengths are about 1.090 1.096 $\AA$, and the TM-Bz distances vary in the range of 1.591 2.510 $\AA$.

Relative stabilities To examine the structural stabilities of $\mathrm{BzVBzTMBz} \quad(\mathrm{TM}=\mathrm{Sc}, \mathrm{Ti}, \mathrm{Cr}-\mathrm{Ni})$, we calculate their binding energies $\left(\mathrm{E}_{\mathrm{b}}\right)$ defined as:

\section{$\mathrm{E}_{\mathrm{b}}=-[\mathrm{E}(\mathrm{V})+\mathrm{E}(\mathrm{TM})+3 \mathrm{E}(\mathrm{Bz})-\mathrm{E}(\mathrm{BzVBzTMBz})]$}

Here, E[-] denotes the total energy of BOSCs, Bz molecules or TM atoms, respectively. As displayed in Table 1, the binding energies are all quite large $(>8.6 \mathrm{eV})$, indicating that they are all thermodynamically stable. Moreover, the binding energies vary in the range of $8.6 \mathrm{eV} \sim 10.8 \mathrm{eV}$, showing that the stabilities of BOSCs have a clear dependence on the metal elements. Of which, BzVBzTiBz has the largest binding energy of $11.755 \mathrm{eV}$, while $\mathrm{BzVBzMnBz}$ and $\mathrm{BzVBzNiBz}$ have the relatively smaller binding energies around $8.6 \mathrm{eV}$.

The element-dependence stabilities of the BzVBzTMBz clusters can be understood from their different bonding characteristic between $\mathrm{TM}$ and $\mathrm{Bz}$ ligand. The frontier orbitals (LUMO down to HOMO-10) are displayed in Fig. 1 and in Fig. S3 in 
Table 1. The point Group symmetry (PGS), total magnetic moment (M), HOMO-LUMO Gap $(\Delta)$, binding energies $\left(E_{b}\right)$, energy difference of second higher spin states with respect to the lowest energy spin-state configurations $(\Delta \mathrm{E})$, distances $\left(\mathrm{R}_{\mathrm{V}-\mathrm{Bz}}, \mathrm{R}_{\mathrm{TM}-\mathrm{Bz}}\right)$ of transition metal atoms to the mass center of their facing $\mathrm{Bz}$ molecules, of the BzVBzTMBz (TM= Sc, Ti, Cr-Ni ) clusters.

\begin{tabular}{cccccccc}
\hline System & PGS & $\mathrm{M} / \mu_{\mathrm{B}}$ & $\Delta / \mathrm{eV}$ & $\mathrm{E}_{\mathrm{b}} / \mathrm{eV}$ & $\Delta \mathrm{E} / \mathrm{eV}$ & $\mathrm{R}_{\mathrm{V}-\mathrm{Bz}} / \AA$ & $\mathrm{R}_{\mathrm{TM}-\mathrm{B} z} / \AA$ \\
\hline $\mathrm{BzVBzScBz} / \AA$ & $\mathrm{C}_{6 \mathrm{~V}}$ & 2 & 0.401 & 10.714 & 0.060 & $1.691,1.655$ & $1.924,1.903$ \\
$\mathrm{BzVBzTiBz}$ & $\mathrm{C}_{6 \mathrm{~V}}$ & 1 & 0.457 & 11.755 & 0.055 & $1.638,1.705$ & $1.798,1.727$ \\
$\mathrm{BzVBzCrBz}$ & $\mathrm{C}_{6 \mathrm{~V}}$ & 3 & 1.001 & 9.745 & 0.280 & $1.637,1.701$ & $1.635,1.577$ \\
$\mathrm{BzVBzMnBz}$ & $\mathrm{C}_{6 \mathrm{~V}}$ & 2 & 0.322 & 8.608 & 0.330 & $1.664,1.714$ & $1.738,1,591$ \\
$\mathrm{BzVBzFeBz}$ & $\mathrm{C}_{6 \mathrm{~V}}$ & 3 & 0.666 & 9.711 & 0.042 & $1.654,1.677$ & $1.724,1.559$ \\
$\mathrm{BzVBzCoBz}$ & $\mathrm{C}_{1}$ & 2 & 0.606 & 10.465 & 0.110 & $1.699,1.702$ & $2.057,1.736$ \\
$\mathrm{BzVBzNiBz}$ & $\mathrm{C}_{1}$ & 1 & 1.358 & 8.601 & 1.350 & $1.675,1.680$ & $1615,2.510$ \\
\hline
\end{tabular}
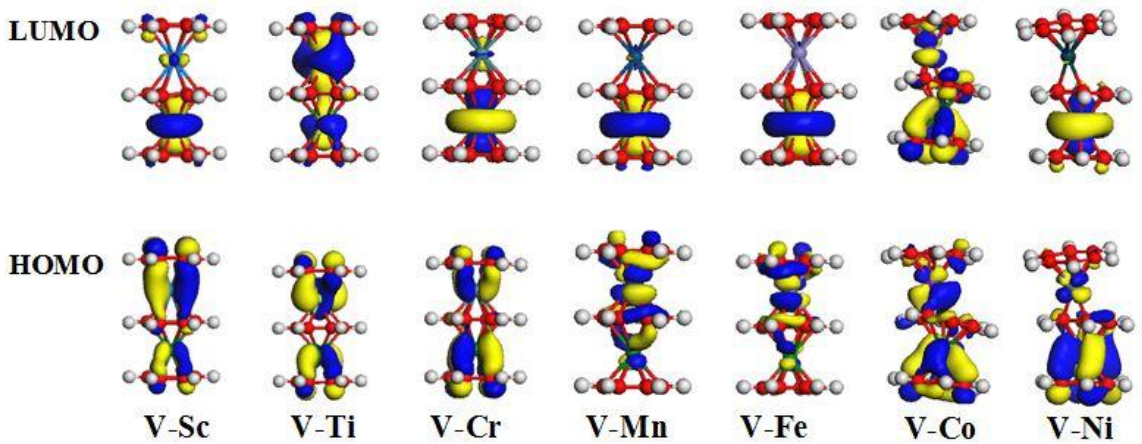

Figure 1. (a) (g) HOMOs and LUMOs orbitals of BzVBzTMBz (TM=Sc, Ti, Cr-Ni) clusters.

Supporting Information. Except $\mathrm{BzVBzCoBz}$, the LUMOs of BzVBzTMBz are nonbonding orbitals derived from the $\mathrm{dz}^{2}$ orbital of TM (including $\mathrm{V}$ ) atoms. Different bonding nature is found in their HOMOs: in the case of $\mathrm{TM}=\mathrm{Sc}, \mathrm{Ti}, \mathrm{Cr}$, they are bonding orbitals derived from the $\mathrm{d}_{\mathrm{xy}}$ or $\mathrm{d}_{\mathrm{x} 2-\mathrm{y} 2}$ orbitals of $\mathrm{TM}$ atoms, while in the case of $\mathrm{TM}=\mathrm{Mn}$ and $\mathrm{Fe}$, their HOMOs are anti-bonding ones and mainly from $\mathrm{d}_{\mathrm{xz}}$ or $\mathrm{d}_{\mathrm{yz}}$ orbitals of TM atoms. As for $\mathrm{BzVBzCoBz}$, its HOMOs and LUMOs are a bit more complicated with significant hybridization of $\mathrm{d}_{\mathrm{xz}}\left(\mathrm{d}_{\mathrm{yz}}\right)$ orbitals of Co and $\mathrm{d}_{\mathrm{x} 2-\mathrm{y} 2}$ orbital of $\mathrm{V}$. Similar bonding features can also be seen in the HOMOs of BzVBzNiBz. The complicated orbital characters of $\mathrm{BzVBzCoBz}$ and $\mathrm{BzVBzNiBz}$ are derived from their large structural deformation. Moreover, we identify eight bonding orbitals for $\mathrm{BzVBzTMBz}(\mathrm{TM}=\mathrm{Sc}, \mathrm{Ti}, \mathrm{Cr}, \mathrm{Co})$, whereas six bonding orbitals and two anti-bonding orbitals are detected for the cases of BzVBzTMBz (TM=Mn, Ni) (Fig. S3 in Supporting Information). The more bonding orbitals of the former four clusters account for the relatively high stability.

Magnetic properties The magnetic moments of the BzVBzTMBz clusters vary in the range of $1 \sim 3 \mu_{B}$ sensitive to the metal elements (see Table 1 and Fig.
2), showing that the bimetallic benzene sandwich clusters can provide more freedom to manipulate the magnetic properties of the clusters. We take BzVBzTMBz (TM=Ti, Mn, Cr) for example to deeply understand their magnetic behaviors (see Fig 2c). Under the crystal field of $\mathrm{Bz}$ ligand, the five degenerate $d$ orbitals of TM atom are split into a singly degenerate $\mathrm{a}_{1}\left(d_{\mathrm{z} 2}\right)$ orbital, two sets of doubly degenerate $\mathrm{e}_{1}\left(d_{\mathrm{yz}}, d_{\mathrm{xz}}\right)$ and $\mathrm{e}_{2}\left(d_{\mathrm{xy}}, d_{\mathrm{x} 2-\mathrm{y} 2}\right)$ orbitals. In the case of BzVBzTiBz, four electrons from Ti atoms equally occupy one degenerate spin-up $\left(\mathrm{e}_{2}\right)$ and spin-down $\left(\mathrm{e}_{2}\right)$ orbitals, contributing zero magnetic moment; while for $\mathrm{V}$, three electrons occupy one singlet $a_{1}$ orbital and one degenerate $e_{2}$ orbital in the spin-up channel, and the other two occupy one $e_{2}$ orbital in the spin-down channel, as a result, the unpaired $\mathrm{dz}^{2}$ electron induces $1 \mu_{\mathrm{B}}$ magnetic moment for BzVBzTiBz. Similarly, for BzVBzMnBz, two unpaired electrons from $\mathrm{Mn}$ and $\mathrm{V}$ atom are found to occupy one degenerate $\mathrm{e}_{1}$ orbital and singlet $\mathrm{a}_{1}$ orbital in the spin-up channel, respectively, which is responsible for total magnetic moment of $2 \mu_{\mathrm{B}}$. And for $\mathrm{BzVBzCrBz}$, one unpaired electron from $\mathrm{V}$ atom is identified to occupy one $\mathrm{a}_{1}$ spin-up orbital, and the other two unpaired ones from $\mathrm{Cr}$ atoms are on one $\mathrm{a}_{1}$ 
and $\mathrm{e}_{1}$ spin-up orbitals, respectively, as a result, the total magnetic moment of $\mathrm{BzVBzCrBz}$ is $3 \mu_{\mathrm{B}}$. Furthermore, to examine the spin stability of the BzVBzTMBz clusters, we calculate their energy differences between FM ground states and second higher energy spin states in Table $\mathbf{1}$ and Fig. 2a.
These energy differences for the BOSCs are around $0.055 \mathrm{eV} \sim 1.350 \mathrm{eV}$, strikingly larger than the value of $0.004 \mathrm{eV} \sim 0.009 \mathrm{eV}$ in pure $\mathrm{V}_{2} \mathrm{Bz}_{3} \quad[13,14,16]$, indicating that mixing different metal atom to $\mathrm{V}_{\mathrm{n}} \mathrm{Bz}$ can effectively enhance the spin stabilities of BOCSs.

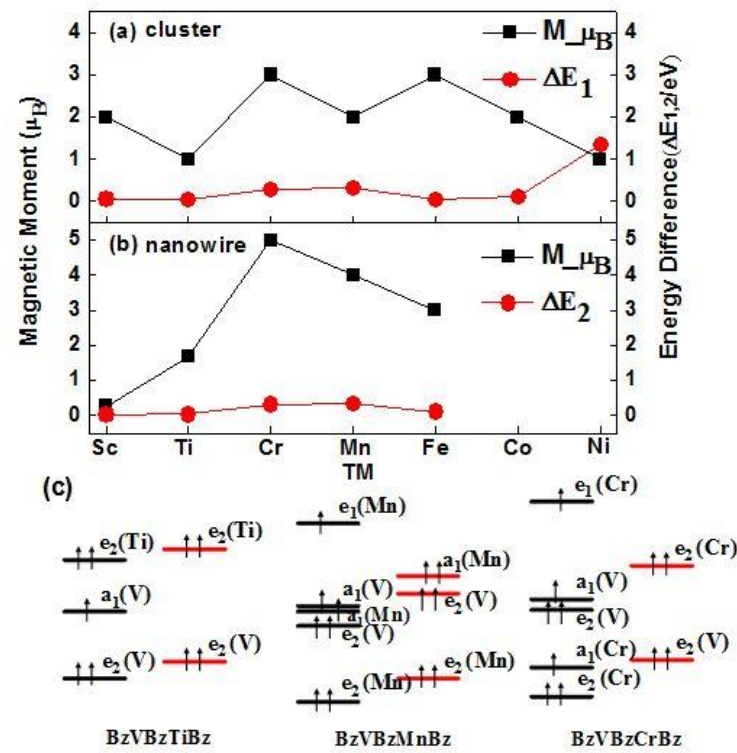

Figure 2. Magnetic moments $\left(\mu_{\mathrm{B}}\right)$ and energy differences between the ground states and the second-lowest energy spin states (or AFM states) of BzVBzTMBz clusters $\left(\Delta \mathrm{E}_{1}\right)(\mathrm{TM}=\mathrm{Sc}, \mathrm{Ti}, \mathrm{Cr}-\mathrm{Ni})(\mathrm{a})$ and $[\mathrm{BzVBzTM}]_{\infty}$ $(\mathrm{TM}=\mathrm{Sc}, \mathrm{Ti}, \mathrm{Cr}-\mathrm{Fe})\left(\Delta \mathrm{E}_{2}\right)$ nanowires per unit (b); (c) The energy orbitals of BzVBzTMBz (TM=Ti, Mn, Cr), the black lines respects the spin up electrons and the red lines are for the spin down electrons.

\subsection{Infinite $[\mathrm{BzVBzTM}]_{\infty}(\mathrm{TM}=\mathrm{Sc}, \mathrm{Ti}, \mathrm{Cr}, \mathrm{Mn}, \mathrm{Fe})$ nanowires.}

Considering the structural deformation of $\mathrm{BzVBzCoBz}$ and $\mathrm{BzVBzNiBz}$, we only explore the structural, electronic and magnetic properties of the other five 1D BOSMWs, [BzVBzTM $]_{\infty}(\mathrm{TM}=\mathrm{Sc}, \mathrm{Ti}$, $\mathrm{Cr}-\mathrm{Fe}$ ) (see Table 2) with eclipsed sandwich configurations. The related information of their higher-energy states is presented in Fig. 2(b) and Table S1 in the Supporting Information. Similarly, to evaluate the stability of the nanowires, we calculate their binding energy per unit cell by following equation: $\quad E_{b}=-\{E(V)+E(T M)$ $\left.+2 \mathrm{E}(\mathrm{Bz})-\mathrm{E}\left([\mathrm{BzVBzTM}]_{\infty}\right)\right\}$, where $\mathrm{E}[\cdot]$ is the total energy of the wires, $\mathrm{Bz}$ molecule and $\mathrm{TM}$ atoms, respectively. Similar with the BOSCs, the relative stability of these BOSMWs varies with metal elements. Of which, $[\mathrm{BzVBzSc}]_{\infty}$ has the largest binding energy of $10.87 \mathrm{eV}$, while $[\mathrm{BzVBzMn}]_{\infty}$ has the smallest one, $5.98 \mathrm{eV}$ (see Table 2). Furthermore, to examine the true magnetic ground states, we also carefully calculate the energy difference of ferromagnetic and antiferromagnetic states of their supercells (see Fig.S1 in the Supporting Information), and our results show that most BOSMWs are FM except $[\mathrm{BzVBzTi}]_{\infty}$ exhibiting AFM coupling. Of which, the magnetic moment is as high as $5 \mu_{\mathrm{B}}, 4 \mu_{\mathrm{B}}$ and $3 \mu_{\mathrm{B}}$ per unit cell for $[\mathrm{BzVBzCr}]_{\infty},[\mathrm{BzVBzMn}]_{\infty}$ and $[\mathrm{BzVBzFe}]_{\infty}$, respectively (see Table 2 ).

The intriguing magnetic properties can be well understood from the electron occupation of their energy bands (see Fig. 3 and Fig. S4). Taking $[\mathrm{BzVBzCr}]_{\infty}$ as example (Fig. 3a), we identify six orbitals that are mainly comprised of $\mathrm{Cr} 3 d$ electrons at the Gamma point of Brillouin Zone (BZ). Five of them fill a singlet $a_{1}$ orbital, two sets of the doubly degenerate $\mathrm{e}_{1}$ and $\mathrm{e}_{2}$ orbitals on the majority manifold, and the other one occupies one minority $e_{2}$ orbital, resulting in four electrons being unpaired. On the other hand, five $3 d$ electrons from $\mathrm{V}$ atom are found to occupy two majority $d$ orbitals $\left(\mathrm{a}_{1}, \mathrm{e}_{2}\right)$ and one minority $e_{2}$ orbital, leading to one electron unpaired. Thus, the total magnetic moment of $[\mathrm{BzVBzCr}]_{\infty}$ is as high as $5 \mu_{\mathrm{B}}$ per unit cell. Similar analysis can also be made for other wires. 
Table 2. Point group symmetry (PGS), lattice constant $c$, the distance of transition metal atoms to the center of its nearby $\mathrm{Bz}$ rings $\left(\mathrm{R}_{\mathrm{V}-\mathrm{Bz}} / \mathrm{R}_{\mathrm{TM}-\mathrm{Bz}}\right)$, binding energy $\mathrm{E}_{\mathrm{b}}$, energy differences between the $\mathrm{FM}$ and AFM/FIM states $(\Delta \mathrm{E})$, total magnetic moment $\left(\mathrm{M}_{\mathrm{t}}\right)$ per unit cell, and electronic ground states $(\mathrm{GS})$ of the BOSMWs.

\begin{tabular}{lllllllll}
\hline System & $\mathrm{c} / \AA$ & $\mathrm{PGS}$ & $\mathrm{R}_{\mathrm{V}-\mathrm{Bz}} / \AA$ & $\mathrm{R}_{\mathrm{TM}-\mathrm{Bz}} /$ & $\mathrm{E}_{\mathrm{b}} / \mathrm{eV}$ & $\Delta \mathrm{E} / \mathrm{eV}$ & $\mathrm{M}_{\mathrm{t}} / \mu_{\mathrm{B}}$ & GS \\
\hline $\mathrm{V}-\mathrm{Sc}$ & 7.18 & $\mathrm{C}_{6 \mathrm{v}}$ & 1.674 & 1.916 & 10.87 & 0.005 & 0.28 & FM Metal \\
$\mathrm{V}-\mathrm{Ti}$ & 7.01 & $\mathrm{C}_{6 \mathrm{v}}$ & 1.693 & 1.811 & 9.32 & -0.013 & 1.67 & AFM Metal \\
$\mathrm{V}-\mathrm{Cr}$ & 7.26 & $\mathrm{C}_{6 \mathrm{v}}$ & 1.722 & 1.909 & 7.91 & 0.319 & 5 & FM HM \\
$\mathrm{V}-\mathrm{Mn}$ & 7.03 & $\mathrm{C}_{6 \mathrm{v}}$ & 1.674 & 1.841 & 5.98 & 0.398 & 4 & FM HM \\
$\mathrm{V}-\mathrm{Fe}$ & 6.94 & $\mathrm{C}_{6 \mathrm{v}}$ & 1.688 & 1.782 & 7.00 & 0.049 & 3 & FM HM \\
\hline
\end{tabular}
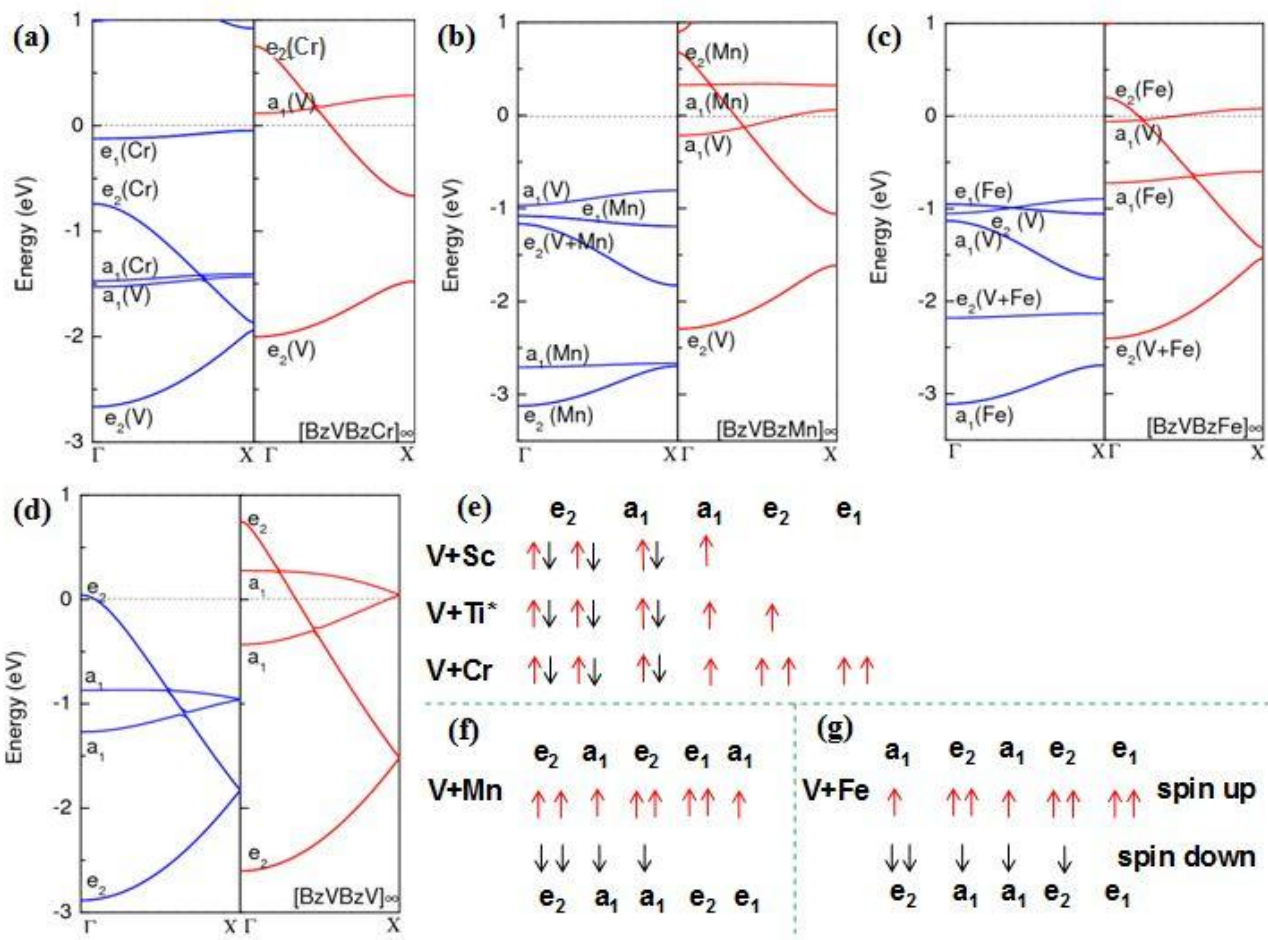

Figure 3. (a-d) Band structures of $[\mathrm{BzVBzTM}]_{\infty}(\mathrm{TM}=\mathrm{Cr}, \mathrm{Mn}, \mathrm{Fe}, \mathrm{V}) \mathrm{HM}$ nanowires; (e-g) Valence electron occupation of TM atoms at the Gamma point of $\mathrm{BZ}$ of $[\mathrm{BzVBzTM}]_{\infty}(\mathrm{TM}=\mathrm{Ti}, \mathrm{Cr}-\mathrm{Fe}) \mathrm{BOSMWs}$.

Besides, the benzene-ligand BOSMWs also show rich electronic properties. Among which, $[\mathrm{BzVBzSc}]_{\infty}$ and $[\mathrm{BzVBzTi}]_{\infty}$ are metals (see Fig. $\mathbf{S 4}$ in Supporting information). Most interestingly, $[\mathrm{BzVBzCr}]_{\infty}$, $[\mathrm{BzVBzMn}]_{\infty}$ and $[\mathrm{BzVBzFe}]_{\infty}$ are found to be $\mathrm{HMs}$ (see Fig. 3a-3c), e.g., it is semiconducting (SC) on one spin channel while it is metallic on the opposite channel. These HMs have $100 \%$ spin polarization at the Fermi level and are potential candidates for spintronics devices. Furthermore, the three HM wires are found to be robust FM HMs, the HM gaps (defined as the difference between the Fermi level and the topmost occupied band in the semiconductor channel) of which are $0.03 \mathrm{eV}, 0.78 \mathrm{eV}$ and $0.93 \mathrm{eV}$ for $[\mathrm{BzVBzCr}]_{\infty}, \quad[\mathrm{BzVBzMn}]_{\infty}$ and $[\mathrm{BzVBzFe}]_{\infty}$, respectively, significantly larger in comparison with that of $[\mathrm{VBz}]_{\infty}$ (nearly zero, see Fig. 3d) [10-14]. Furthermore, to understand the electronic properties of these $[\mathrm{BzVBzTM}]_{\infty}(\mathrm{TM}=\mathrm{Sc}, \mathrm{Ti}, \mathrm{Cr}-\mathrm{Fe})$ nanowires, we carefully examine the valence electron occupation of TM atoms in the studied $[\mathrm{BzVBzTM}]_{\infty}$ wires at the Gamma points in the whole BZ (Fig. 3e-3g). Interestingly, we find that: (1) The valence electrons of TM atoms first occupy three spin-up orbitals $\left(a_{1}\right.$, $\mathrm{e}_{2}$ ) and three spin-down orbitals $\left(\mathrm{a}_{1}, \mathrm{e}_{2}\right)$; (2) When more valence electrons are present $(\mathrm{N}>6, \mathrm{~N}$ is the total valence electrons of two TM atoms), they turn back to fill the spin-up channel until another five orbitals are fully occupied; (3) If $\mathrm{N}>11$, the excess electrons begin to fill the spin-down orbitals. This 
electron filling rule is very similar with those of $\left[\mathrm{CpTM}_{1} \mathrm{CpTM}_{2}\right]_{\infty}$ wires[27] except of no significant magnitude of charge transferring from TM to $\mathrm{Bz}$.

Finally, we make an comparison on the different electronic properties between $\left[\mathrm{BzTM}_{1} \mathrm{BzTM}_{2}\right]_{\infty}$ and $\left[\mathrm{CpTM}_{1} \mathrm{CpTM}_{2}\right]_{\infty}$ (see Table 3) BOSMWs. In the case of $\mathrm{TM}_{1}=\mathrm{V}$ and $\mathrm{TM}_{2}=\mathrm{Ti}, \mathrm{V}, \mathrm{Mn}$, similar electronic properties are found [10-14,19,27], e.g. it is metal at $\mathrm{TM}_{2}=\mathrm{Ti}$ and $\mathrm{HM}$ at $\mathrm{TM}_{2}=\mathrm{V}, \mathrm{Mn}$. In contrast, different electronic behaviors are identified for other BOSMWs listed in Table 3. For example, the Cp ligand $[\mathrm{CpVCpTM}]_{\infty}\left(\mathrm{TM}_{2}=\mathrm{Cr}, \mathrm{Fe}\right),[\mathrm{CpTiCpTM}]_{\infty}$ $\left(\mathrm{TM}_{2}=\mathrm{Mn}, \mathrm{Fe}\right)$ and $[\mathrm{CpCrCpMn}]_{\infty}$ are semiconducting (SC), while their corresponding Bz-ligand analogues are HMs. Moreover, $[\mathrm{CpTiCpCr}]_{\infty}$ is $\mathrm{HM}$, while $[\mathrm{BzTiBzCr}]_{\infty}$ is metal. Above results indicate that the TM-Bz combinations seem to be more half metallic or metallic (see Table 3) than their $\mathrm{Cp}$ based complexes. To get a deep understanding of their different electronic behavior, we plotted the band structures as well as the iso-surfaces of electron charge density differences (CDD) of $[\mathrm{CpVCpCr}]_{\infty}$ (semiconductor) and $[\mathrm{BzVBzCr}]_{\infty}$ (half metal) wires in Fig. 4. For $[\mathrm{CpVCpCr}]_{\infty}$, the $\mathrm{e}_{1}$ band from $\mathrm{Cr}$ atom in the majority channel is empty and above the Fermi Level, while it is completely occupied and under the Fermi Level in $[\mathrm{BzVBzCr}]_{\infty}$, which is consistent with the fact that two electrons need to be transferred from TM atoms to two $\mathrm{Cp}$ ligands. Moreover, the electron CDD around $\mathrm{TM}$ and $\mathrm{Cp}$ in $[\mathrm{CpVCpCr}]_{\infty}$ is a bit higher than that in $[\mathrm{BzVBzCr}]_{\infty}$, indicating significant electron transfer in the $\mathrm{Cp}$ ligand complexes and the metal-ligand interaction is more ionic for $[\mathrm{CpVCpCr}]_{\infty}$ than $[\mathrm{BzVBzCr}]_{\infty}$, which is in good agreement with previous reports $[19,25,27]$. Therefore, the electronic behaviors of these organometallic sandwich nanowires have a clear dependence on the bonding mechanism between TM atoms and organic ligands, which deserves further investigation in future. Seen from Fig. $\mathbf{4 a}, \mathbf{b}$, in correspondence with the $[\mathrm{CpVCpCr}]_{\infty}$, the conducting bands in $[\mathrm{BzVBzCr}]_{\infty}$ are more close to the Fermi level, particularly, the $\mathrm{e}_{2}$ band in the minority channel cross the Fermi level, leading it to be HM.

Table 3. Comparision of Electronic Properties between $\left[\mathrm{BzTM}_{1} \mathrm{BzTM}_{2}\right]_{\infty}$ and $\left[\mathrm{CpTM} \mathrm{CpTM}_{2}\right]_{\infty}$ wires.

\begin{tabular}{llllllllll}
\hline System & V-Ti & V-V & V-Cr & V-Mn & V-Fe & Ti-Cr & Ti-Mn & Ti-Fe & Cr-Mn \\
\hline$\left[\mathrm{BzTM}_{1} \mathrm{BzTM}_{2}\right]_{\infty}$ & Metal & $\mathrm{HM}^{[10-14]}$ & $\mathrm{HM}$ & $\mathrm{HM}$ & $\mathrm{HM}$ & Metal & $\mathrm{HM}$ & $\mathrm{HM}$ & $\mathrm{HM}$ \\
$\left.\mathrm{CpTM}_{1} \mathrm{CpTM}_{2}\right]_{\infty}$ & Metal $^{[27]}$ & $\mathrm{HM}^{[19]}$ & $\mathrm{SC}^{[27]}$ & $\mathrm{HM}$ & $\mathrm{SC}^{[24,25]}$ & $\mathrm{HM}^{[27]}$ & $\mathrm{SC}^{[27]}$ & $\mathrm{SC}^{[25]}$ & $\mathrm{SC}^{[27]}$
\end{tabular}

(a)
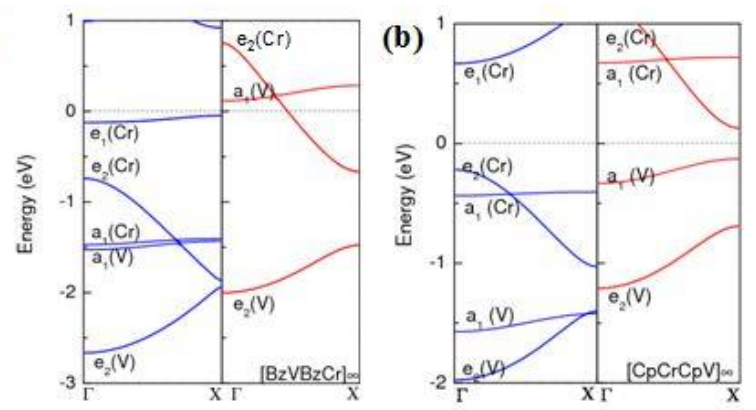

(c)

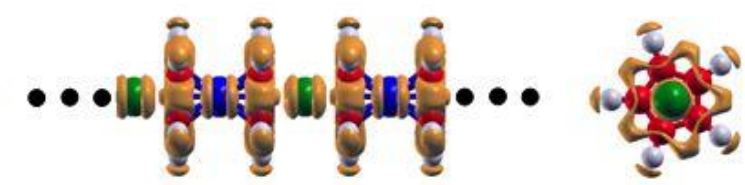

(d)

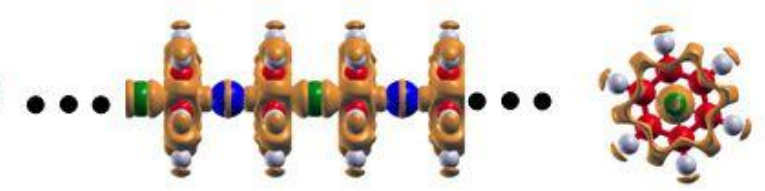

Figure 4. (a, b)Band structures of $[\mathrm{CpVCpCr}]_{\infty}$ and $[\mathrm{BzVBzCr}]_{\infty}$. (c, d)Iso-surfaces of electron charge density difference for $[\mathrm{CpVCpCr}]_{\infty}$ and $[\mathrm{BzVBzCr}]_{\infty}$, the isovalue is used as $0.06 \mathrm{e} / \AA^{3}$. 


\section{Conclusion}

In summary, we have extensively investigated the structural, electronic and magnetic properties of one-dimensional bimetallic organic multi-decker sandwich clusters, BzVBzTMBz, and their infinite BOSMWs, [BzVBzTM $]_{\infty}(\mathrm{TM}=\mathrm{Sc}, \mathrm{Ti}, \mathrm{Cr}-\mathrm{Ni})$ by using spin-polarized density functional theory approach. Most BzVBzTMBz clusters and 1D nanowires are found to be thermodynamically stable. And the magnetic moments of the BzVBzTMBz clusters have a clear dependence on the TM elements. Except $[\mathrm{BzVBzSc}]_{\infty}$ and $[\mathrm{BzVBzTi}]_{\infty}$ are metals, $[\mathrm{BzVBzCr}]_{\infty}, \quad[\mathrm{BzVBzMn}]_{\infty}$ and $[\mathrm{BzVBzFe}]_{\infty}$ are found to be robust ferromagnetic $\mathrm{HMs}$, of which, the $\mathrm{HM}$ gaps of $[\mathrm{BzVBzMn}]_{\infty}$ and $[\mathrm{BzVBzFe}]_{\infty}$ are significantly larger than that of monometal $[\mathrm{VBz}]_{\infty}$. Furthermore, comparing with their $\left[\mathrm{CpTM}_{1} \mathrm{CpTM}_{2}\right]_{\infty}$ analogues, the covalent interaction between TM and benzene molecules make $[\mathrm{BzVBzTM}]_{\infty}$ more half metallic or metallic, which can be helpful in predicting novel organometallic HM materials.

\section{Acknowledgement}

The work is supported by the National Nature Science Foundation of China (No.1110424). The authors thank the computational resource at Department of Physics, YZU.

Supporting Information. Side views of single-cell $[\mathrm{BzVBzTM}]_{\infty}$ and super-cell [BzVBzTMBzVBzTM] $\infty$ configurationss per unit cell (red dotted line) and supercell (double unit cell, blue dotted line) representations (Figure S1). Lowest energy structures of $\mathrm{BzVBzTMBz}(\mathrm{TM}=\mathrm{Sc}-\mathrm{Ni}$ ) clusters (Figure $\mathrm{S} 2$ ), frontier orbitals $\left(\mathrm{HOMO} \mathrm{HOMO}_{-10}\right.$ ) of BzVBzTMBz $(\mathrm{TM}=\mathrm{Sc}-\mathrm{Ni}$ ) (Figure S3), band structures of $[\mathrm{BzVBzSc}]_{\infty}$ and $[\mathrm{BzVBzTi}]_{\infty}$ (Figure S4), the energy differences of different spin states of $[\mathrm{BzVBzTM}]_{\infty}$ $(\mathrm{TM}=\mathrm{S}, \quad \mathrm{Ti} \quad \mathrm{Cr}-\mathrm{Fe})$ wires (Table S1). www.ccaasmag.org/chem_2012/vol2/Zhang_BzVBz_ Supp.pdf.

\section{References}

[1]Hoshino, K.; Kurikawa, T.; Takeda, H.; Nakajima, A.; Kaya, K. J. Phys. Chem. 1995, 99, 3053-3055. http://dx.doi.org/10.1021/j100010a013

[2] Kurikawa, T.; Takeda, H.; Hirano, M.; Judai, K.; Arita, T.; Nagao, S.; Nakajima, A.; Kaya, K. Organometallics 1999, 18, 1430-1438. http://dx.doi.org/10.1021/om9807349

[3] Nakajima, A.; Kaya, K. J. Phys. Chem. A 2000, 104, 176-191. http://dx.doi.org/10.1021/jp9927303

[4] Pandey, R.; Rao, K. B.; Jena, P.; Blanco, M. A. J. Am. Chem. Soc. 2001, 123, 3799-3808.

http://dx.doi.org/10.1021/ja0035452
[5] Kandalam, A. K.; Rao, P. Jena, B. K.; Pandey, R. J. Chem. Phys. 2004, 120, 10414.

http://dx.doi.org/10.1063/1.1738632

[6] Miyajima, K.; Nakajima, A.; Yabushita, S.; Knickelbein, M. B.; Kaya, K. J. Am. Chem. Soc. 2004, 126, 13202-13203.

http://dx.doi.org/10.1021/ja046151+

[7] Wang, J.; Acioli, P. H.; Jellinek, J. J. Am. Chem. Soc. 2005, 127, 2812-2813.

http://dx.doi.org/10.1021/ja043807q

[8] Mokrousov, Y.; Atodiresei, N.; Bihlmayer, G.; Gel, S. Int. J. Quantum Chem. 2006, 106, 3208-3213. http://dx.doi.org/10.1002/qua.21139

[9] Miyajima, K.; Yabushita, S.; Knickelbein, M. B.; Nakajima, A. J. Am. Chem. Soc. 2007, 129, 8473-8480. http://dx.doi.org/10.1021/ja070137q

[10] Rahman, M. M.; Kasai, H.; Dy, E. S. Jpn. J. Appl. Phys. 2005, 44, 7954.

http://dx.doi.org/10.1143/JJAP.44.7954

[11] Xiang, H. J.; Yang, J. L.; Hou, J. G.; Zhu, Q. S. J. Am. Chem. Soc. 2008, 128, 2310-2314. http://dx.doi.org/10.1021/ja054751i

[12] Maslyuk, V. V.; Bagrets, A.; Meded, V.; Arnold, A.; Evers, F.; Brandbyge, M.; Bredow, T.; Mertig, I. Phys. Rev. Lett. 2006, 97, 097201. http://dx.doi.org/10.1103/PhysRevLett.97.097201

[13] Mokrousov, Y.; Atodiresei, N.; Bihlmayer, G.; Gel, S.; Blugel, S. Nanotechnology 2007, 18, 495402.

http://dx.doi.org/10.1088/0957-4484/18/49/495402

[14] Weng, H. M.; Ozaki, T.; Terakura, K. J. Phys. Soc. Jpn. 2008, 77, 014301.

http://dx.doi.org/10.1143/JPSJ.77.014301

[15] Zhang, X.; Wang, J. J. Phys. Chem. A 2008, 112, 296-304. http://dx.doi.org/10.1021/jp076647o

[16] Zhang, X.; Wang, J. J. Phys. Chem. A 2010, 114, 2319-2323. http://dx.doi.org/10.1021/jp907834v

[17] Kua, J.; Tomlin, K. M. J. Phys. Chem. A 2006, 110, 11988-11994. http://dx.doi.org/10.1021/jp065341z

[18] Hosoya, N.; Takegami, R.; Suzumura, J.; Yada, K.; Koyasu, K.; Miyajima, K.; Mitsul, M.; Knickelbein, M. B.; Yabushita, S.; Nakajima, A. J. Phys. Chem. A 2005, 109, 9-12.

http://dx.doi.org/10.1021/jp0452103

[19] Shen, L.; Yang, S. W.; Ng, M. F.; Ligatchev, V.; Zhou, L.; Feng, Y. J. Am. Chem. Soc.2008, 130, 13956-13960. http://dx.doi.org/10.1021/ja804053a

[20] Zhou, L.; Yang, S.; Ng, M.; Sullivan, M. B.;Tan, V. B. C.; Shen, L. J. Am. Chem. Soc. 2008, 130, 4023-4027. http://dx.doi.org/10.1021/ja7100246 
[21] Mallajosyula, S.; Parida, P.; Pati, S. J. Mater. Chem. 2009, 19, 1761-1766.

http://dx.doi.org/10.1039/b810089f

[22] Zhu, L.; Wang, J. J. Phys. Chem. C 2009, 113, 8767-8771. http://dx.doi.org/10.1021/jp9018298

[23] Nagao, S.; Kato, A.; Nakajima, A. J. Am. Chem. Soc. 2010, 122, 4221-4222.

http://dx.doi.org/10.1021/ja994506x

[24] Wang, L.; Cai, Z.; Wang, J.; Lu, J.; Luo, G.; Lai, L.; Zhou, J.; Qin, R.; Gao, Z.; Yu, D.; Li, G.; Mei, W. N. Nano Lett. 2008, 8, 3640-3644.

http://dx.doi.org/10.1021/nl8016016

[25] Zhang, X.; Wang, J.; Gao, Y.; Zeng, X. ACS Nano. 2009, 3, 537-545. http://dx.doi.org/10.1021/nn800794c

[26] Wu, J. C.; Wang, X. F.; Zhou, L.; Da, H. X.; Lim, K. H.; Yang, S. W.; Li, Z. Y. J. Phys. Chem. C 2009, 113, 7913-7916. http://dx.doi.org/10.1021/jp902718h

[27] Zhang, X.; Yang, S.W.; Tian, Z.; Zhu, L.Y. ; Wang, J. J. Phys. Chem. C 2011, 115, 2948-2953. http://dx.doi.org/10.1021/jp109253a
[28] Kresse, G.; Hafner, J. Phys. Rev. B 1993, 48, 13115. http://dx.doi.org/10.1103/PhysRevB.48.13115

[29] Kresse, G.; Furthmüller, J. Comput. Mater. Sci. 1996, $6,15-50$.

http://dx.doi.org/10.1016/0927-0256(96)00008-0

[30] Perdew, J. P.; Burke, K.; Ernzerhof, M. Phys. Rev. Lett. 1996, 77, 3865.

http://dx.doi.org/10.1103/PhysRevLett.77.3865

[31] Blochl, P. E. Phys. Rev. B 1994, 50, 17953. http://dx.doi.org/10.1103/PhysRevB.50.17953

[32] Kresse, G.; Joubert, D. Phys. Rev. B 1999, 59, 1758. http://dx.doi.org/10.1103/PhysRevB.59.1758

[33]DMOL is a density functional theory program distributed by Accelrys, Delley, Inc. B. J. Chem. Phys. 1990, 92, 508. J. Chem. Phys.2000, 113, 7756. http://dx.doi.org/10.1063/1.458452 http://dx.doi.org/10.1063/1.1316015

[34] Rao, B. K.; Jena, P. J. Chem. Phys. 2002, 116 , 1343-1349.

http://dx.doi.org/10.1063/1.1429245 